\title{
El audiovisual The first monday in may y su utilización como recurso para la mejora del aprendizaje. Puesta en valor $y$ aplicación en el Máster en Gestión Cultural de la Universitat Politècnica de València
}

\begin{abstract}
Francisca Ramón Fernándeza, Vicente Cabedo Mallol, María Emilia Casar Furióc, Vicent Giménez Chornet ${ }^{\mathrm{d}}$, Cristina Lull Noguera ${ }^{\mathrm{e}}$, Juan Vicente Oltra Gutiérrez ${ }^{\mathrm{f}}$ y Desamparados Soriano Soto ${ }^{g}$

aProfesora titular. Departamento de Urbanismo, Universitat Politècnica de València,

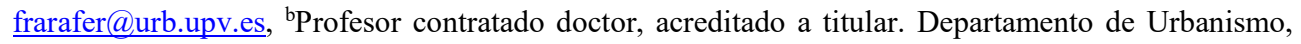

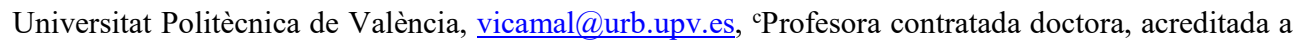
titular. Departamento de Urbanismo, Universitat Politècnica de València, macafu@urb.upv.es, ${ }^{d}$ Profesor titular. Departamento de Comunicación Audiovisual, Documentación e Historia del Arte,

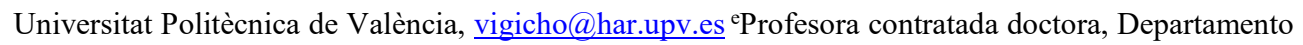

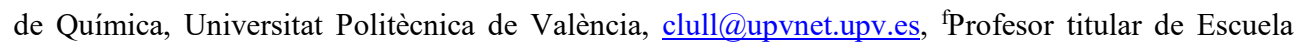
Universitaria. Departamento de Organización de Empresas, Universitat Politècnica de València, jvoltra@omp.upv.es, ${ }^{\mathrm{g}}$ Catedrática. Departamento de Producción Vegetal, Universitat Politècnica de València, asoriano@prv.upv.es
\end{abstract}

\begin{abstract}
It presents the innovation and results obtained from applying the audiovisual "The first monday in may" as a resource to improve learning. Through the visisionado of the same and the answer of a survey we can observe that the students of the Master in Cultural Management improve their learning on contents that can observe in the documentary and to discuss on its application in the cultural industries. The results obtained show a favorable influence on the use of audiovisual media in the classroom as a tool for improving the learning of concepts, as well as the ethical and professional commitment to the issues raised.
\end{abstract}

Keywords: Improved learning, teaching innovation, Master in Cultural Management, The first monday in may, audiovisual, application, results

\footnotetext{
Resumen

Se presenta la innovación y resultados obtenidos de aplicar el audiovisual "The first monday in may" como recurso para mejorar el aprendizaje. A través del visionado del mismo y la contestación de una encuesta podemos observar que los alumnos del Máster en Gestión Cultural mejoran su aprendizaje sobre contenidos que pueden observar en el documental y discutir sobre su aplicación en las industrias culturales. Los resultados obtenidos muestran una
} 
influencia favorable a la utilización de los medios audiovisuales en el aula como herramienta para la mejora de aprendizaje de conceptos, así como el compromiso ético y profesional a las cuestiones planteadas.

Palabras clave: Mejora aprendizaje, innovación docente, Máster en Gestión Cultural, The first Monday in may, audiovisual, aplicación, resultados

\section{Introducción}

Siguiendo en la línea de innovación docente del grupo RETAJUDOCA (Recursos tecnológicos para el aprendizaje jurídico, la documentación y la comunicación audiovisual) de la Universitat Politècnica de València en la que desarrollamos materiales para la mejora docente en ciencias sociales y jurídicas a través de la utilización de audiovisuales (Cabedo et al., 2017 y 2018 y Ramón et al., 2018a, b y c), mostramos en el presente trabajo el resultado de aplicar un audivisual con notas de humor crítico, que se enmarca dentro del PIME que desarrollamos durante los cursos académicos 2018-2020, referente al "Diseño de materiales docentes basados en recursos audiovisuales de humor para la mejora del aprendizaje y su evaluación en ciencias sociales y jurídicas”.

Se expondrán los resultados obtenidos en la experiencia que hemos desarrollado en el presente curso académico 2018-2019, tras utilizar el audiovisual The first monday in may en la titulación del Máster en Gestión Cultural, en concreto en la asignatura "Marco jurídico aplicado a la protección del patrimonio y los museos" con la finalidad de observar el grado de aprendizaje y su mejora por parte de los alumnos, tras su visionado y contestación a una encuesta de preguntas abiertas.

\section{Objetivos}

Se ha diseñado una encuesta específica para valorar la mejora del aprendizaje de conceptos por parte de los alumnos con los siguientes objetivos:

a) Utilización de un audiovisual atractivo, real y sobre un tema de actualidad, en la línea de los PIMEs que desarrolla el grupo.

b) Incrementar el espíritu crítico del alumnado determinando la ética, legalidad y aplicación en nuestro país de una experiencia desarrollada en Estados Unidos.

c) Validar la experiencia de innovación docente como viable y si se mejora el aprendizaje de conceptos previos explicados por el profesorado.

d) Observar si el alumnado capta el tono irónico y de humor negro que se desprende del audiovisual en cuanto lo relacione con el logro que se pretende.

\section{La utilización del audiovisual The first monday in may y su utilización como recurso para la mejora del aprendizaje}

Se ha elegido el audiovisual The first monday in may (2016) dirigido por Andrew Rossi que se centra en la denominada Gala del "The Metropolitan Museun of Art de New York (MET)", acto benéfico organizado por la directora de Vogue América, Anna Wintour, con la finalidad de recaudar fondos para el museo. Se pretende así superar las anteriores exposiciones que se 
han realizado en el museo, con una exposición sobre China, en concreto "China Through the Looking Glass".

La razón de elección de dicho audiovisual radica en varios factores ligados a la Gala MET que nos interesan para el desarrollo de la innovación docente:

a) Realización de una actividad cultural dentro del espacio destinado a un Museo.

b) Finalidad específica de recaudar fondos para el Museo.

c) Exposición de carácter internacional con intención de superar las anteriores exposiciones del Museo.

d) Actividad lúdica con participación de los más importantes nombres del mundo de la moda y la cultura.

e) Audiovisual que muestra notas de humor sobre cómo hacer posible lo imposible.

Tratamos de utilizar el audiovisual para mejorar el aprendizaje teniendo en cuenta que está destinado a un perfil de alumnos muy determinado, en el que queremos desarrollar su espíritu crítico y mostrar una actividad real que plantea dudas éticas, de gestión y de financiación.

Las competencias de la asignatura orientadas a la futura actividad profesional son las siguientes:

1. Que sean capaces de integrar conocimientos y enfrentarse a la complejidad de formulación de juicios. Deberán incluirse reflexiones sobre responsabilidad sociales y éticas aplicables.

2. Capacidad de aplicar conocimientos y resolución de problemas en nuevos entornos y relacionados con contextos multidisciplinares.

3. Comprensión de conocimientos para desarrollar un aporte original en una actividad de investigación.

4. Conocimiento de la normativa jurídica aplicable a la gestión cultural.

La competencia transversal que se trabaja y que es punto de control es la de "Pensamiento crítico", con la que se pretende que el alumnado desarrolle una actitud propia y crítica sobre los fundamentos que soportan las acciones y juicios tanto de sí mismo como de los demás.

Esta competencia en el futuro profesional de un gestor cultural es fundamental para desarrollar sus actividades en entornos complejos, y por ello, la utilización del presente audiovisual al estar íntimamente relacionado con la gestión de un museo, nos permite observar la capacidad del alumnado para reflexionar y argumentar, deslindar entre lo aceptable y lo denostable, de un actividad profesional en un museo.

La utilización de audiovisuales en el aprendizaje constituye una herramienta muy idónea para la elaboración de casos prácticos soportados previamente con un fragmento o parte de una película, serie o documental, y ha sido ya puesto en valor por la doctrina en el ámbito de la innovación docente (Cabedo et al., 2016; Ramón et. Al., 2016a y b, y Oltra, 2016). 


\section{Desarrollo de la innovación y su aplicación en el Máster en Gestión Cultural}

En el Máster de Gestión Cultural los alumnos adquieren conocimientos relacionados con la gestión de los museos, y con el marco jurídico relacionado con la defensa del patrimonio. En los últimos años los museos están priorizando la atracción de la audiencia frente al rol tradicional de custodia del patrimonio. Esto obliga a los responsables de los museos a comprender tanto el papel de custodia como la necesidad de atraer un mayor número de visitantes (Gilmore, Rentschler, 2002). La amplia competencia en la oferta cultural obliga a los profesionales de los museos a tomar interés por los servicios que presta su organización frente a la gestión y conservación de los objetos custodiados. En este sentido, centrarse en la gestión del marketing de museos conlleva explorar la influencia de sus competidores para poder generar más visitantes, de este modo los museos podrían justificar su dependencia financiera de los Gobiernos (Komarac, Ozretic-Dosen, Skare, 2017). Las operaciones tradicionales de las instituciones museísticas, que giran en torno a preservar, investigar, exhibir e interpretar los objetos de su patrimonio, se enfrentan al desafío de no poder atraer a su público potencial, con el reto de atraer a una generación más joven y aumentar la accesibilidad (Chaotzu Wang, Quo-Ping Lin, 2018).

Para que los alumnos reflexionaran sobre el evento que se realiza en el MET, como actividad para generar tanto más visitantes como más financiación, se les formularon previamente las siguientes cuestiones a las que tenía que estar atentos durante la proyección del documental:

1. ¿Cómo surgió la idea del evento?

2. ¿Este tipo de financiación se podría aplicar en España?

3. ¿Qué tecnologías utilizan en el MET para la gestión?

4. ¿Es éticamente aceptable el comportamiento de los organizadores/trabajadores del evento?

Estas cuestiones se trasladaron posteriormente a un debate en el aula, donde los alumnos pudieron discutir sobre los beneficios de crear eventos en los museos, sobre las diferencias de financiación entre el mundo anglosajón y el contexto español, sobre el uso de tecnologías en la gestión del evento, y sobre el comportamiento de los trabajadores reflexionando entre un contexto laboral de Estados Unidos y un contexto funcionarial español.

\section{Resultados obtenidos}

En la actividad docente participaron siete alumnos. Consideramos que es una muestra poco representativa y que no se puede generalizar a ninguna población los resultados. Sin embargo, sí que nos aporta información sobre la utilización de audiovisuales en el aula, y entendemos que en el futuro con una muestra mayor podermos obtener resultados más aplicables. Después de una hora de debate en el aula se les pasó un cuestionario para ver el interés de la actividad, en una escala de cinco valores.

Como se ve en la Figura 1, unas tres cuartas partes de los alumnos consideran que el empleo de elementos audiovisuales en el aula es totalmente positivo para poder debatir un caso práctico. 


\section{Gráfico 1: ¿Es interesante el empleo en el aula de elementos audiovisuales para debatir el problema de casos prácticos?}

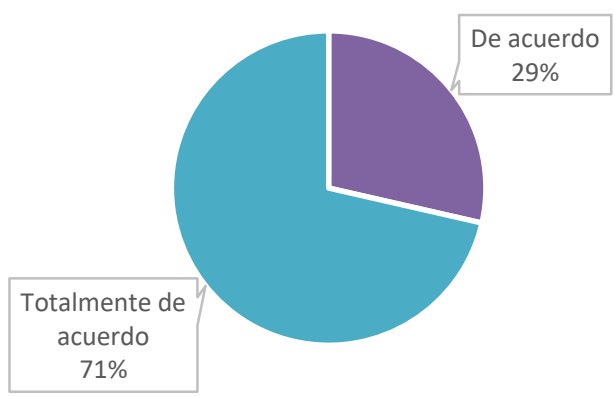

$$
\begin{aligned}
& \text { - Totalmente en desacuerdo } \\
& \text { - Indiferente } \\
& \text { - Totalmente de acuerdo }
\end{aligned}
$$$$
\text { - En desacuerdo }
$$$$
\text { - De acuerdo }
$$

Fig. 1. Respuestas obtenidas sobre la pregunta ¿Es interesante el empleo en el aula de elementos audiovisuales para debatir el problema de casos prácticos?. Fuente: elaboración propia.

Aunque el audiovisual se centra en el mundo de la moda se pueden extraer elementos jurídicos relacionados con normativas de financiación, protección del patrimonio o derecho internacional. Todos los alumnos detectaron elementos jurídicos, pero solo el $43 \%$ ha estado totalmente de acuerdo (Figura 2).

\section{Gráfico 2: ¿Habéis detectado elementos jurídicos en el vídeo?}

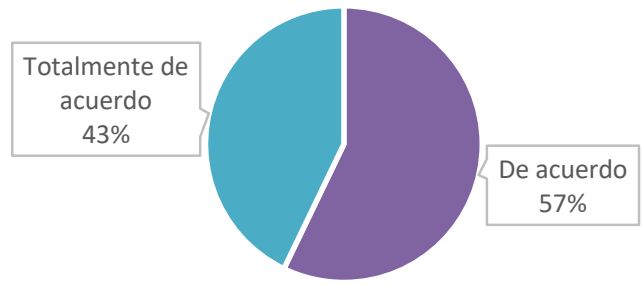

\footnotetext{
- Totalmente en desacuerdo

- Indiferente

- Totalmente de acuerdo
}

- En desacuerdo

Fig. 2. Porcentaje de respuestas en relación con la pregunta ¿Habéis detectado elementos jurídicos en el vídeo?. Fuente: elaboración propia.

Más de la mitad de los alumnos está totalmente de acuerdo que la visualización del video más el debate surgido después en el aula ayuda a comprender los contenidos dados en la asignatura "Marco jurídico aplicado a la protección del patrimonio y a los museos", y los alumnos restantes están de acuerdo en esta actividad (Figura 3 ). 


\section{Gráfico 3: La actividad realizada, visualización del vídeo más el debate en clase cos ha ayudado a comprender mejor los conocimientos de la asignatura?}

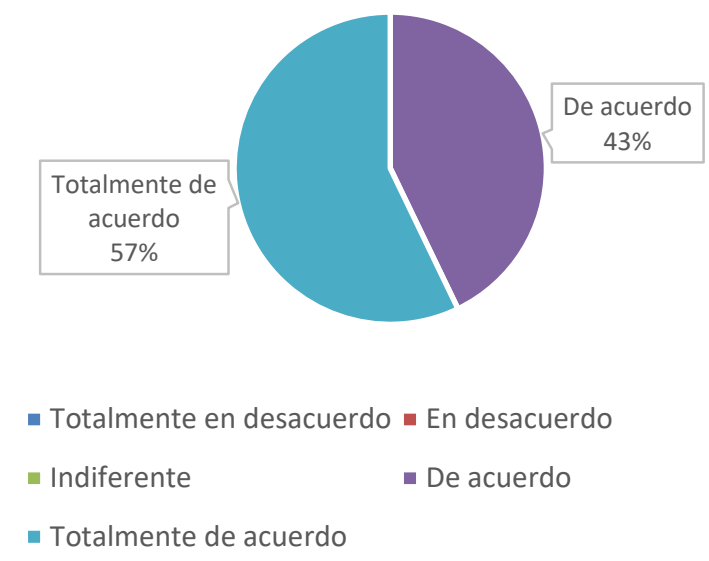

Fig. 3. Porcentaje obtenido sobre la consulta si la actividad realizada, visualización del vídeo más debate en clase ayuda a la comprensión de los conocimientos de la asignatura. Fuente: elaboración propia.

La asignatura de Marco Jurídico es punto de control de la competencia "Pensamiento crítico". En clase se ha explicado qué significa esta competencia y qué acciones se pueden realizar para ejercitarla, entre otras el análisis de un caso práctico y debatir sobre el mismo. En este sentido casi tres cuartas parte del alumnado considera que la actividad ha resultado útil para ejercitar la competencia de pensamiento crítico (Figura 4).

\section{Gráfico 4: ¿El vídeo os ha resultado útil para el desarrollo de la competencia transversal de "Pensamiento crítico"?}

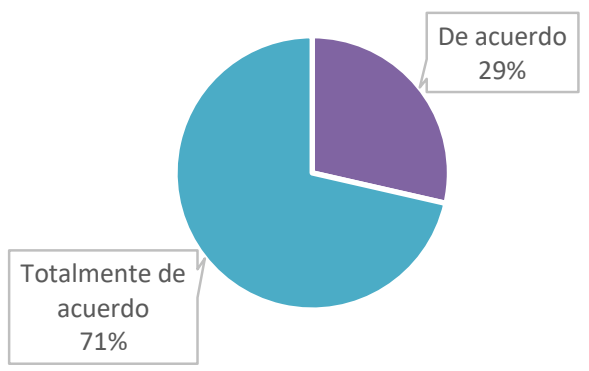

- Totalmente en desacuerdo - En desacuerdo

- Indiferente

- De acuerdo

- Totalmente de acuerdo

Fig. 4. Porcentaje obtenido en la pregunta sobre si el vídeo ha resultado útil para el desarrollo de la competencia transversal de "Pensamiento crítico". Fuente: elaboración propia.

Aunque la asignatura no es punto de control de la competencia "Responsabilidad ética y profesional" hemos tratado en el debate los comportamientos éticos y profesionales de los 
trabajadores del MET y de las distintas personas encargadas de realizar el evento. La mayoría de los alumnos están de acuerdo que esta actividad es provechosa, y un $43 \%$ la considera como totalmente de acuerdo (Figura 5).

\section{Gráfico 5: El vídeo y el debate en clase ¿ha ayudado a aplicar la competencia transversal de responsabilidad ética y profesional?}
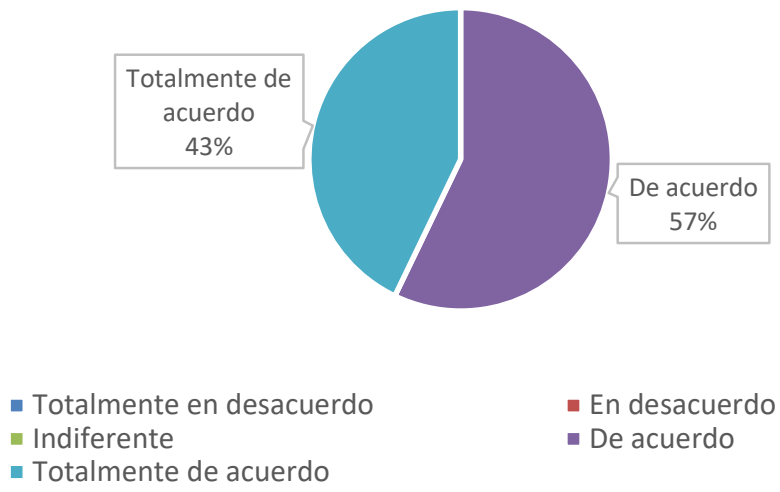

Fig. 5. Porcentaje de respuesta sobre la consulta acerca de si el vídeo y el debate en clase ayudan a la aplicación de la competencia transversal de responsabilidad ética y profesional. Fuente: elaboración propia.

Cuando se imparte la asignatura de Marco Jurídico en el master de Gestión Cultural desconocemos si los alumnos la consideran importante en el conjunto de conocimientos adquiridos en dicho máster. La actividad ha revelado que para una gran mayoría de los alumnos les ha supuesto un estímulo valorar la importancia del conocimiento de las leyes, especialmente para el posterior desarrollo de su profesión como gestores culturales (Figura $6)$.

\section{Gráfico 6: ¿La actividad os ha supuesto un estímulo para valorar la importancia del marco jurídico en la gestión cultural?

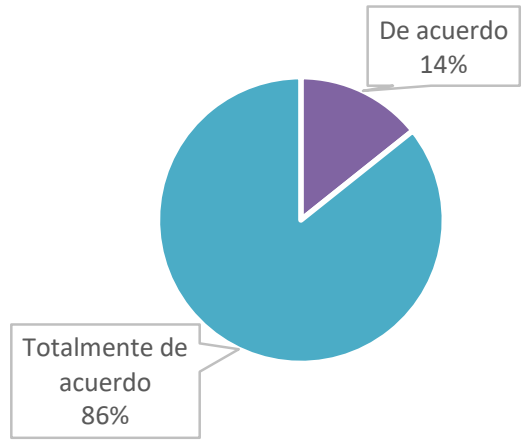 \\ - Totalmente en desacuerdo \\ - En desacuerdo \\ - Indiferente \\ - De acuerdo \\ - Totalmente de acuerdo}


El audiovisual The first Monday in may y su utilización como recurso para la mejora del aprendizaje. Puesta en valor y aplicación en el Máster en Gestión Cultural de la Universitat Politècnica de València

Fig. 6. Respuesta sobre la consulta acerca de si la actividad ha supuesto un estímulo para valorar la importancia del marco jurídico en la gestión cultural. Fuente: elaboración propia.

Como se puede observar en el Figura 7, todos los alumnos han valorado muy positivamente la actividad de visualización del video "El primer lunes de mayo", que trata sobre la organización de un evento y exposición sobre la moda en el MET de Nueva York.

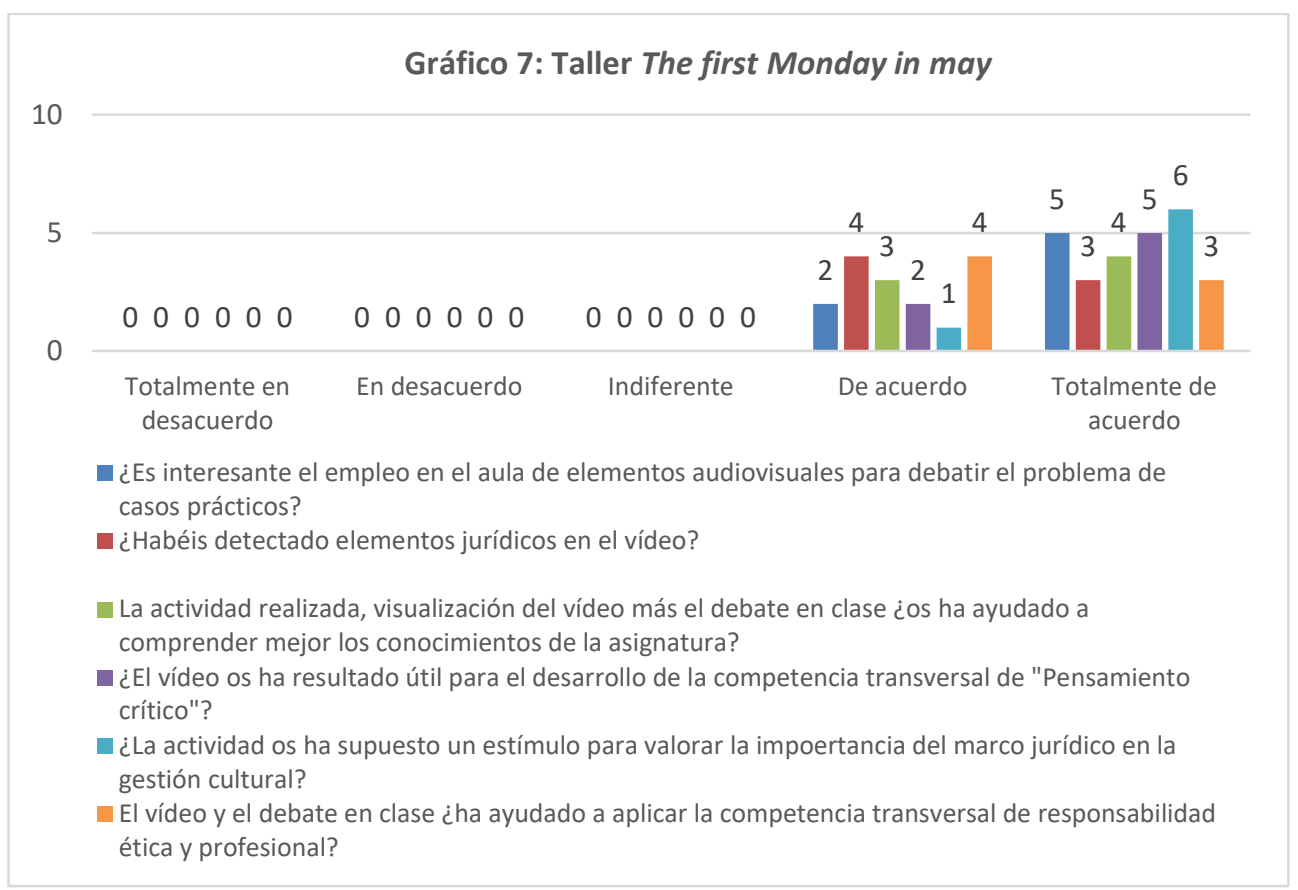

Fig. 7. Valoración de los alumnos sobre la actividad realizada en el taller. Fuente: elaboración propia.

\section{Conclusiones}

Para terminar de valorar esta actividad los alumnos tenían la opción voluntaria de responder a una pregunta abierta para opinar sobre el taller realizado en relación a su aprendizaje. A algunos les ha sorprendido la mayor facilidad de poder recaudar fondos en Estados Unidos a diferencia de España, a otros las cuestiones éticas en la organización del trabajo, o las diferencias en el sistema laboral entre una gestión privada y una pública, como en España, y en general han valorado el taller positivamente como forma de analizar la gestión de un evento desde dentro de una institución cultural.

De esta experiencia, a pesar de ser un número poco significativo de alumnado sobre el que hemos realizado la actividad de innovación, podemos indicar que ha cubierto los objetivos planteados, y que la utilización del audiovisual en el aprendizaje ha permitido una consolidación de los conceptos, así como ha facilitado la evaluación de la competencia transversal punto de control de la asignatura.

\section{Agradecimientos}

Trabajo realizado en el marco del Proyecto de Innovación y Mejora Educativa (PIMEs) "Diseño de materiales docentes basados en recursos audiovisuales de humor para la mejora 
del aprendizaje y su evaluación en ciencias sociales y jurídicas", presentado en el marco de la convocatoria de Proyectos de Innovación y Mejora Educativa (PIMEs) llevada a cabo en la Universitat Politècnica de València para el curso 2017-2018 obteniendo resolución favorable de la Comisión de Evaluación y Seguimiento de Proyectos de Innovación y Convergencia (CESPIC) en su sesión de 24 de julio de 2018 y concedido por el Vicerrectorado de Estudios y Convergencia Europea de la Universitat Politècnica de València. Años 2018-2020. Investigadora principal: Francisca Ramón Fernández. Investigadores: Vicente Cabedo Mallol, María Emilia Casar Furió, Vicent Giménez Chornet, Cristina Lull Noguera y Juan Vicente Oltra Gutiérrez, Enrique Orduña Malea y Amparo Soriano Soto y en el marco del Proyecto I+D+i «Retos investigación» del Programa estatal de $\mathrm{I}+\mathrm{D}+\mathrm{i}$ orientado a los Retos de la Sociedad del Ministerio de Ciencia, Innovación y Universidades: RTI2018-097354-B-100. «Contratos, transparencia y protección de datos en el mercado digital» (2019-2022). Investigadores Principales: Dr. Javier Plaza Penadés, Catedrático de Derecho Civil, y Dra. Luz M. Martínez Velencoso, Catedrática de Derecho Civil. Universitat de València-Estudi General.

\section{Referencias}

CABEDO MALLOL, V., CASAR FURIÓ, M. E., GIMÉNEZ CHORNET, V., OLTRA GUTIÉRREZ, J. V. y RAMÓN FERNÁNDEZ, F. (2016). Casos prácticos jurídicos basados en series de animación. Francisca Ramón Fernández (coord.). Valencia: Tirant lo Blanch.

CABEDO MALLOL, V., CASAR FURIÓ, M. E., GIMÉNEZ CHORNET, V., LULL NOGUERA, C., OLTRA GUTIÉRREZ, J. V. y RAMÓN FERNÁNDEZ, F. (2017). Casos prácticos jurídicos basados en series de ficción. Francisca Ramón Fernández (coord.). Valencia: Tirant lo Blanch.

CABEDO MALLOL, V., CASAR FURIÓ, M. E., GIMÉNEZ CHORNET, V., LULl NOGUERA, C., OLTRA GUTIÉRREZ, J. V. y RAMÓN FERNÁNDEZ, F. (2018). Casos prácticos jurídicos basados en documentales. Francisca Ramón Fernández (coord.). Valencia: Tirant lo Blanch.

Chaotzu Wang, M., Quo-PIng Lin, J., (2018) "The Future Museum shapes the museum future: A progressive strategy of the National Palace Museum adopting new media art exhibitions as a marketing tool", Arts and the Market, Vol. 8 Issue: 2, pp.168181, https://doi.org/10.1108/AAM-12-2017-0030 [Consulta: 2 de febrero de 2019].

Gilmore, A., RenTSChler, R. (2002) "Changes in museum management: A custodial or marketing emphasis?", Journal of Management Development, Vol. 21 Issue: 10, pp.745-760, https://doi.org/10.1108/02621710210448020 [Consulta: 2 de febrero de 2019].

Komarac, T., Ozretic-Dosen, D., Skare, V. (2017) "Understanding competition and service offer in museum marketing", Academia Revista Latinoamericana de Administración, Vol. 30 Issue: 2, pp.215-230, https://doi.org/10.1108/ARLA-072015-0159 [Consulta: 4 de febrero de 2019]. 
METROPOLITAN MUSEUM OF ART NEW YORK (2019). <https://www.metmuseum.org//> [Consulta: 28 de enero de 2019].

OLTRA GUTIÉRREZ, J. V., RAMÓN FERNÁNDEZ, F., CABEDO MALLOL, V., CASAR FURIÓ, M. E., y GIMÉNEZ CHORNET, V. (2016). "Uso de fragmentos de películas y series como herramientas de innovación docente. Una experiencia con alumnos de informática", en INRED. II Congreso Nacional de Innovación Educativa y Docencia en Red, Universitat Politècnica de València.

RAMÓN FERNÁNDEZ, F., CABEDO MALLOL, V., CASAR FURIÓ, M. E., GIMÉNEZ CHORNET, V., LULL NOGUERA, C. y OLTRA GUTIÉRREZ, J. V. (2016a). "Diseño de casos prácticos jurídicos basados en series de animación. Una innovación docente con soporte audiovisual", en Derecho y TIC: últimas innovaciones docentes, Coordinadores Ana María Delgado García e Ignacio Beltrán de Heredia Ruiz. Barcelona: Huygens editorial, pp. 123-132.

RAMÓN FERNÁNDEZ, F., CABEDO MALLOL, V., CASAR FURIÓ, M. E., GIMÉNEZ CHORNET, V., y OLTRA GUTIÉRREZ, J. V. (2016b). "Utilización de las series de animación como recurso didáctico en la elaboración de casos. Análisis de una experiencia en la innovación docente en la Universitat Politècnica de València", en INRED. II Congreso Nacional de Innovación Educativa y Docencia en Red, Universitat Politècnica de València.

RAMÓN FERNÁNDEZ, F., CABEDO MALLOL, V., CASAR FURIÓ, M. E., GIMÉNEZ CHORNET, V., LULL NOGUERA, C. y OLTRA GUTIÉRREZ, J. V. (2018a). "La utilización de los documentales como herramienta para la evaluación de la competencia transversal CT07. Responsabilidad ética, medioambiental y profesional. Experiencias en la docencia impartida en la Universitat Politècnica de València”, en Derecho y TIC: últimas innovaciones docentes, Coordinadores Ana María Delgado García e Ignacio Beltrán de Heredia Ruiz. Barcelona: Huygens editorial, pp. 193-204.

RAMÓN FERNÁNDEZ, F., CABEDO MALLOL, V., CASAR FURIÓ, M. E., GIMÉNEZ CHORNET, V., LULL NOGUERA, C. y OLTRA GUTIÉRREZ, J. V. (2018b). "La utilización de los documentales como herramienta para la evaluación de la competencia transversal CT07. Responsabilidad ética, medioambiental y professional”, en Jornada de Innovación Docente ETSINF 2018.

RAMÓN FERNÁNDEZ, F., CABEDO MALLOL, V., CASAR FURIÓ, M. E., GIMÉNEZ CHORNET, V., LULL NOGUERA, C., OLTRA GUTIÉRREZ, J. V. y ORDUÑA MALEA, E. (2018c). "El audiovisual Tutankamón como herramienta para la evaluación de la competencia transversal de pensamiento crítico y responsabilidad ética medioambiental y profesional. Una experiencia en la Universitat Politècnica de València”, en IN-RED 2018,. Valencia: Universitat Politècnica de València.

ROSSI, A. (dir.) (2006). First monday in may. <http://www.firstmondayinmay.com/> > [Consulta: 28 de enero de 2019]. 